\title{
The role of high mobility group box 1 protein in acute cerebrovascular diseases (Review)
}

\author{
SHU-WEN MU ${ }^{*}$, YUAN DANG ${ }^{2 *}$, SHOU-SEN WANG ${ }^{1}$ and JIAN-JUN GU ${ }^{3}$ \\ Departments of ${ }^{1}$ Neurosurgery and ${ }^{2}$ Comparative Medicine, Dongfang Affiliated Hospital of Xiamen University, Xiamen \\ University Medical College, Fuzhou, Fujian 350025; ${ }^{3}$ Department of Neuro-interventional Radiology, Zhengzhou \\ University People's Hospital, Henan Provincial People's Hospital, Zhengzhou, Henan 450000, P.R. China
}

Received January 31, 2018; Accepted June 6, 2018

DOI: $10.3892 /$ br.2018.1127

\begin{abstract}
The occurrence and development of acute cerebrovascular diseases involves an inflammatory response, and high mobility group box protein 1 (HMGB1) is a pro-inflammatory factor that is expressed not only in the early-injury stage of disease, but also during the post-repair process. In the initial stage of disease, HMGB1 is released into the outside of the cell to participate in the cascade amplification reaction of inflammation, causing vasospasm, destruction of the blood-brain barrier and apoptosis of nerve cells. In the recovery stage of disease, HMGB1 can promote tissue repair and remodeling, which can aid in nerve function recovery. This review summarizes the biological characteristics of HMGB1, and the role of HMGB1 in ischemic and hemorrhagic cerebrovascular disease, and cerebral venous thrombosis.
\end{abstract}

\section{Contents}

1. Introduction

2. HMGB1

3. HMGB1 and acute cerebrovascular disease

4. Conclusion

Correspondence to: Dr Jian-jun Gu, Department of Neuro-interventional Radiology, Zhengzhou University People's Hospital, Henan Provincial People's Hospital, 7 Weiwu Road, Zhengzhou, Henan 450000, P.R. China

E-mail: gujianjundt@163.com

Dr Shou-sen Wang, Department of Neurosurgery, Dongfang Affiliated Hospital of Xiamen University, Xiamen University Medical College, 156 Xi'erhuanbei Road, Fuzhou, Fujian 350025, P.R. China

E-mail: wshsen@126.com

*Contributed equally

Key words: high mobility group box protein 1, acute ischemic cerebrovascular disease, acute hemorrhagic cerebrovascular disease, cerebral venous sinus thrombosis, inflammation

\section{Introduction}

High mobility group box protein 1 (HMGB1) is the non-chromosome-related group of proteins. It was first isolated in 1973 by Goodwin et al (1), and it is named after its rapid rate of electrophoresis in a polyacrylamide gel. HMGB1 is expressed in the nucleus of almost all eukaryotic cells and is encoded by the human HMGB1 gene (13q12) (2). HMGB1 is involved in stabilizing chromosomal structure in the nucleus, and in regulating the transcription of genes that are critical for maintaining basic life processes. When released from the cell, HMGB1 binds to its specific receptor under specific pathological or physiological conditions, which can mediate multiple inflammatory and autoimmune diseases (3).

In recent years, the high incidence of cerebrovascular disease has markedly affected the lives of patients (4). According to recently released data, in hospitalized patients aged between 55 and 63 years in the United States, the incidence of acute ischemic stroke is $202.5 / 10,000$, the incidence of subarachnoid hemorrhage (SAH) is $11.9 / 10,000$ and the incidence of intracerebral hemorrhage is 22.6/10,000 (4). Although treatment methods have improved over time, treatment remains invasive $(5,6)$. Therefore, it is important to investigate the pathogenesis of cerebrovascular disease and to identify non-invasive treatment methods.

An increasing number of studies have demonstrated that the inflammatory response involving HMGB1 serves an important role in the course of acute cerebrovascular disease. This review summarized the structure, function, receptors and signaling pathways of HMGB1, and retrospectively analyzed the role of HMGB1 in ischemic cerebrovascular disease, hemorrhagic cerebrovascular disease and cerebral venous sinus thrombosis.

\section{HMGB1}

The structure of $H M G B 1$. The sequence and structure of the HMGB1 protein are highly evolutionarily conserved. HMGB1 is composed of 215 amino acids, and has a molecular weight of $25 \mathrm{kDa}$. HMGB1 includes three structural domains: Two relatively rigid DNA binding domains (A and B box) located at the N-terminal, which is termed the HMG box field, and a negatively charged acidic tail comprising 30 glutamic and 
aspartic acids (7,8). The A box is located at the 1-79 loci of the HMGB1 molecular amino acid sequence and the B box is located at the 86-162 loci, and the amino acid homology rate of the two is $>80 \%$. The acidic tail between the B box and the $\mathrm{C}$-terminal is connected by a flexible connection containing 24 amino acids (Fig. 1). Following HMGB1 being released to the outside of the cell, the B box is the main structural functional area that causes inflammation $(7,9)$. The A box has an antagonistic effect on the inflammatory response caused by the B box, and this anti-inflammatory ability is enhanced following the fusion of the acidic C-terminal.

The HMGB1 molecule contains two nuclear localization sequences (NLS), respectively located in the A box (28-44) and the junction area of box B and the C tail (179-185). It also contains three cysteine residues, which are located separately at the 23 and 45 sites of the A box and the 106 locus of box B (Fig. 1) (8). Following stimulation, two cysteine residues can form a disulfide bond, and thus HMGB1 exists as three subtypes, the 'disulfide HMGB1', 'thiol HMGB1' and 'oxidized HMGB1' (10). Disulfide HMGB1 is the main subtype involved in the acute and chronic inflammatory response in the extracellular space and serum, which can further activate macrophages/monocytes to amplify the inflammatory response. The mechanism of HMGB1 is mainly involved in non-inflammatory responses and its mechanism has yet to be elucidated. Thiol HMGB1 can be released early and is able to repair cell damage by recruiting inflammatory cells (11).

Secretion of HMGB1. HMGB1 is secreted by two modes: Passive release and active secretion. The two secretory pathways differ in their molecular mechanism, release kinetics and downstream signaling pathway. Passive release occurs instantaneously upon the destruction of cellular integrity, as HMGB1 is not associated with nuclear DNA in living cells (8).

Under the stimulation of pathogen/microbe-associated molecular patterns and endogenous inflammatory mediators, including tumor necrosis factor (TNF), interleukin-1 (IL-1) and interferon- $\gamma($ IFN- $\gamma)$, macrophages, monocytes, dendritic cells, endothelial cells and other immune cells can actively secrete HMGB1 (8). HMGB1 can also induce its own release through pre-feedback regulation. Neurons, astrocytes, leukemia cells and neuroblastoma cells can also promote the active secretion of HMGB1 (8). Active secretion is much slower than passive release, and can be divided into two steps. To begin with, the HMGB1 in the nucleus is transferred to the cytoplasm through the internuclear pore. This process relies on the Janus kinase-signal transducer and activator of transcription signaling pathway and the super-acetylation of two key lysine residues in NLS (12), preventing HMB1 from entering the cytoplasm and returning to the nucleus, which may aid HMGB1 in accumulating in the cytoplasm. The second stage gradually induces the programmed death of inflammatory cells; alternatively, through secreted lysosomes, the intracellular HMGB1 is released from the cells (13).

Biological functions of HMGB1. Under physiological conditions, HMGB1 is involved in stabilizing chromosomal structure in the nucleus, maintaining gene stability, and induces DNA bending. In this process, box A of the two molecules of HMGB1 can form a special structure successively or

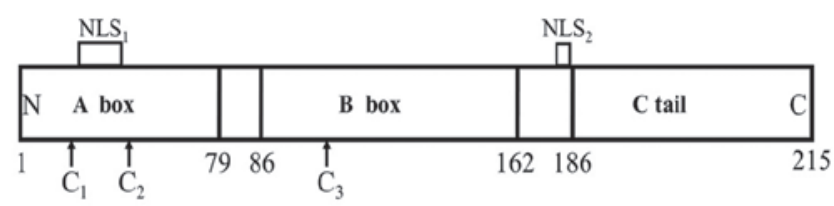

Figure 1. Structure of HMGB1. HMGB1 is comprised of 215 amino acids and has a molecular weight of $\sim 25 \mathrm{kDa}$. HMGB1 includes three structural domains: A box, B box and an acidic tail. It contains two NLS, NLS1 (28-44 loci) and NLS2 (179-185 loci), and three cysteine residues, C1 (23 loci), C2 (45 loci) and C3 (106 loci). HMGB1, high mobility group box B1; NLS, nuclear localization sequences.

simultaneously with DNA, which causes the DNA to bend and reconstruct (14). In addition, HMGB1 can directly participate in the repair process of DNA damage following binding to DNA, namely in nucleotide, base excision, mismatch and double chain fracture repairs (15). The absence of HMGB1 can lead to increasing chromosomal instability (16).

Yanai et al (17), demonstrated that the absence of HMGB1 would weaken the response of the body to the extracellular signals associated with viral invasion, Toll-like receptor (TLR) ligands and PRRs. Therefore, HMGB1 may have a central role in early immunization activities. HMGB1 was one of the first members of its family to be identified. The downstream inflammatory ligands can induce the oligomerization of NOD-like receptor (NLR) and the assembly of inflammatory protein complexes (18). NLR molecules contain a leucine-rich repeat (LRR) domain, which has the functions of a combination of ligands. HMGB1 can also induce cell stress, mediating double-stranded RNA-dependent protein kinase autophosphorylation and promoting inflammatory cascade amplification in the process (19).

The HMGB1 in the cytoplasm can initiate autophagy, which is a self-protective process that removes damaged mitochondria and microbial invasions in the cell by combining with beclin-1 (20).

The HMGB1 signaling pathway. RAGE was the first identified receptor of HMGB1; it is a multifunctional transmembrane receptor of the immunoglobulin superfamily, which is involved in the maintenance of homeostasis and the occurrence of inflammation, and is encoded by a gene on chromosome 6p21.3 (21). When combined with HMGB1, it can activate p38 mitogen-activated protein kinase (MAPK), extracellular signal-regulated kinase 1 (ERK1) and ERK2, which in turn causes phosphorylation and degradation of the inhibitor of nuclear factor $\kappa \mathrm{B}$ (IкB) to activate NF- $\kappa \mathrm{B}$ (22). HMGB1/RAGE can also induce the expression of MAPK, vascular cell adhesion molecule 1 and matrix metalloproteinase (MMP) (23).

The TLR is a member of the type I transmembrane superfamily, and consists of an extracellular LRR structural domain and a Toll/interleukin-1 receptor (TIR) structural domain in the cytoplasm (21). The TLR signaling pathway is divided into MyD88-dependent and MyD88-independent pathways. Following combining with a single ligand, TLR upregulates MyD88 or other adaptive molecules to induce the activation of downstream factors, including NF- $\kappa$ B, MAPK and IFN regulatory factors (24). HMGB1 interacts with 
TLR-2, TLR-4 and TLR-9, triggering the activation of NK- $\kappa$ B and IRF pathways (25). Following the stimulation of TLR2, the downstream signal can be mediated by Racl and PI3K, increasing the adhesion of CD11b/CD18 and intercellular cell adhesion molecule-1 (ICAM-1), and Akt is activated directly by the p65 transcription complex or by the I $\mathrm{B}$ kinase pathway; both of these pathways are able to activate $\mathrm{NF}-\kappa \mathrm{B}$ (22). Following the binding of TLR-4 to HMGB1, the interleukin-1 receptor-related kinase 1 can be phosphorylated, thereby activating the downstream signaling molecule $\mathrm{NF}-\kappa \mathrm{B}$, and promoting MAPK by phosphorylating JNK, ERK, p38 and $\mathrm{I} \kappa \mathrm{B}(26)$. TLR9, normally located in the endoplasmic reticulum, can be transferred to the early endosomes in an HMGB1-dependent manner (27). TLR9 in endosomes and/or lysosomes can identify CpG DNA binding to HMGB1, and can then mediate $\mathrm{NF}-\kappa \mathrm{B}$ and its downstream inflammatory response (28). In recent years, it has been demonstrated that the combination of HMGB1 and TLR5 can also rely on MyD88 to activate the downstream signal (29).

$\mathrm{C}-\mathrm{X}-\mathrm{C}$ chemokine receptor 4 (CXCR4) is a member of the $\mathrm{G}$ protein-coupled receptor family, which has a low expression in normal tissues, but a significantly higher expression in tumor tissues (21). Recent studies have demonstrated that CXCR4 is also involved in inflammatory responses, and that thiol HMGB1 can form complexes with CXCL12 and activate CXCR4 to promote the production of inflammatory cells and cytokines (30).

\section{HMGB1 and acute cerebrovascular disease}

Acute ischemic cerebrovascular disease. Worldwide, ischemic cerebrovascular disease is the leading cause of disability and the third leading cause of mortality, and therefore is a heavy social and economic burden (31). Following cerebral ischemia, neuroinflammation and stress serve important roles in the pathogenesis of the disease (31). The inflammatory response in ischemic stroke consists of two stages: The early stage of destruction of the nerve tissue and the late stage of organizational reconstruction.

Following acute ischemic stroke, damaged brain tissue can release HMGB1, recruiting a variety of pro-inflammatory cytokines and chemokines, and increasing adhesion molecule expression, further activating brain cells and immune cells (32). Following cerebral ischemia, astrocytes and endothelial cells may be the early targets of HMGB1, which can be activated in this process. The former can directly transmit signals to neurons and blood vessels, and the latter upregulates ICAM-1 expression, recruiting immune cells into the ischemic area (33). The destruction of the blood brain barrier (BBB) is an important stage of ischemic brain injury, involving multiple cytokines. The activation of MMPs and the expression of various proteases results in the decomposition of the $\mathrm{BBB}$, exacerbating leukocyte extravasation (34). HMGB1 can increase vascular permeability and promote BBB decomposition (35). Anti-HMGB1 antibody can inhibit the morphological and functional changes in the BBB induced by HMGB1 (36). Tsukagawa et al (32) demonstrated that quantitative serum HMGB1 levels could be used to evaluate the prognosis of ischemic stroke and may be more accurate than the existing evaluation methods.
Ischemic reperfusion injury can further aggravate functional metabolic disorders and structural damage in ischemic tissues. Apoptosis is strictly regulated by the MAPK family, and the c-Jun N-terminal kinase (JNK), ERK1/2 and p38 protein family in ischemia reperfusion injury is activated (37). The continuous activation of MAPK is associated with the death or apoptosis of neurons in the post-stroke stage of ischemic stroke (37). A study by Gong et al (38), demonstrated that glycyrrhizin can be used as a HMGB1 inhibitor to inhibit the JNK and p38 pathways in rats. Umahara et al (39), revealed that in the chronic stages of cerebral infarction, certain macrophages that were located in the ischemic region were positive for HMGB1. They hypothesized that there may be two reasons for this. One is that the HMGB1-associated inflammatory response in chronic cerebral infarction develops from acute cerebral infarction. Alternatively, HMGB1-positive macrophages may induce autophagy in the area of chronic ischemic injury, possibly due to the fact that HMGB1 can maintain autophagy (20).

In the recovery phase of ischemic stroke, HMGB1 may promote brain remodeling. Chen et al (40) and Wu et al (41), demonstrated that IL-6 and vascular endothelial growth factor (VEGF) mediate the reactive astrocyte release of HMGB1, and participate in the angiogenesis and neurogenesis in the late phase of stroke, promoting brain remodeling and neurological function recovery. Brain remodeling was inhibited following the administration of HMGB1 inhibitors. These studies have aided in improving the prognosis of ischemic stroke at different stages.

\section{Acute hemorrhagic cerebrovascular disease}

Intracranial aneurysm and SAH. Intracranial aneurysms are pathological local dilations caused by changes in local intracranial vessels. Among the various causes of SAH, spontaneous aneurysm rupture is the most common and requires attention. Zhang et al (42), demonstrated that HMGB1 was highly expressed on ruptured and unruptured aneurysm walls and, compared with the latter, the former had a higher level of expression. However, there is no significant association between the size of the aneurysm and the expression level of HMGB1. Through double immunofluorescence staining, Chalouhi et al (43) demonstrated that HMGB1 was expressed in the nucleus of smooth muscle cells, macrophages, lymphocytes and endothelial cells; these cells were involved in the remodeling of the aneurysm wall. $\mathrm{NF}-\kappa \mathrm{B}$ is highly expressed in the aneurysm wall, and the formation of the aneurysm is hindered by the application of $\mathrm{NF}-\kappa \mathrm{B}$ inhibitors (44). The incidence of intracranial aneurysms is associated with atherosclerosis and HMGB1 is involved in the formation of atheromatous plaques; following endothelial cell injury, activated NF- $\mathrm{NB}$ induces the activation of leukocyte adhesion molecules and a variety of cytokines, including HMGB1, and these signals can collect macrophages in the wall of blood vessels. Macrophages immersed in vessel walls can transform into foam cells and release HMGB1 again, forming a positive feedback inflammatory pathway (45). It has been demonstrated that in the formation and development of intracranial aneurysms, HMGB1 mediates the inflammatory response and participates in the formation of atherosclerotic plaques; as a result, the vascular wall is thickened or narrowed, and vascular remodeling increases the risk of rupture. Therefore, inhibition of HMGB1 may alleviate atheromatous plaque formation and reduce the risk of aneurysm rupture. 
SAH is a life-threatening central nervous system disease. Cerebral vasospasm is one of the most important causes of the high morbidity and mortality of SAH. Previous study have demonstrated that $30-70 \%$ of patients with aneurysms and SAH will have vasospasm (46). It has been observed that this pathophysiological process is associated with inflammatory reactions, including leukocyte recruitment, infiltration and activation. Following SAH, HMGB1 participates in the inflammatory response and serves an important role in apoptosis and vascular spasm (47). Zhao et al (48), revealed that the artery endothelial cells and smooth muscle cells in the damaged brain region following $\mathrm{SAH}$ were activated to stimulate the secretion of HMGB1, which may promote intracranial arterial spasms. Umahara et al (39), conducted autopsies of patients with SAH and revealed that an HMGB1-like immunoreaction was observed in the cytoplasm of vascular smooth muscle cells in the hematoma. HMGB1 could not only promote the occurrence of cerebral vasospasm, but also increase the gene and protein expression levels of RAGE in neurons and microglia around the hematoma, and the number of microglia was also significantly increased. As a main downstream factor of RAGE, the main p65 subunit of NF- $\kappa B$ was significantly increased, indicating that RAGE promoted the activation of NF- $\kappa \mathrm{B}$ at the early stage of SAH (49). Resveratrol, as an inhibitor of HMGB1, can relieve this pathophysiological change; the reason for this is that resveratrol may be involved in SAH-induced neuronal apoptosis, brain edema and nerve injury via inhibition of the HMGB1-mediated TLR4/MyD88/NF-kB pathway in the early stage of SAH (50). Clinically, patients with aneurysmal SAH may develop cerebral vasospasm and delayed cerebral ischemia, increased expression of HMGB1 during the course of disease, increased cerebral vasospasms and eventually an increased risk of cerebral infarction (51). Therefore, HMGB1 rapidly interacts with nerve cells and glial cells following $\mathrm{SAH}$, and participates in cerebral vasospasm and apoptosis.

In the recovery phase of SAH, Tian et al (52) suggested that HMGB1 can promote neurological recovery and blood vessel regeneration via RAGE mediation, which further demonstrated that HMGB1 serves different roles at different disease stages.

Intracranial hemorrhage (ICH). ICH accounts for $\sim 15 \%$ of all stroke cases and has a mortality rate close to $50 \%$ (53). Even patients who survive ICH often experience severe disability (53). The reaction that follows ICH is a complex process and involves a series of pathophysiological reactions, including excitotoxicity, free radical injury and inflammatory reactions (54). Hematoma can cause inflammatory reactions in the surrounding tissue, and can activate glial cells and neurons to aggravate cerebral injury (55). Following acute ICH, HMGB1 is released into the extracellular space by the damaged cell and, as a proinflammatory cytokine, it immediately causes a downstream inflammatory reaction (56), leading to brain damage, affecting neurobehavioral functioning, increasing the permeability of the BBB and aggravating brain edema (57). It has been reported that an anti-HMGB1 antibody can improve brain injury and nerve function defects following ICH in rats (58). In this process, HMGB1 triggers three specific downstream receptors,

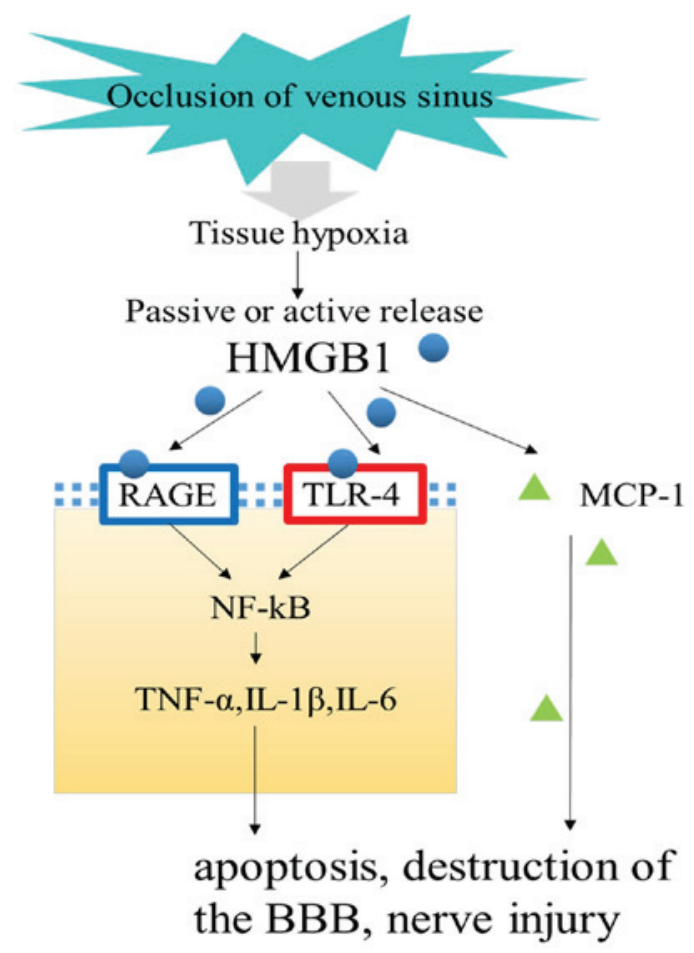

Figure 2. Signaling pathway of HMGB1 in cerebral venous sinus thrombosis Following thrombosis, the pressure in the veins and capillaries is higher and blood return is limited, leading to tissue hypoxia. Subsequently, damaged tissue can actively or passively release HMGB1, triggering downstream inflammatory responses, participating in BBB disruption, apoptosis and nerve damage, and further aggravating brain damage. HMGB1, high mobility group box B1; BBB, blood brain barrier; TNF- $\alpha$, tumor necrosis factor $\alpha$; IL, interleukin; MCP-1, monocyte chemoattractant protein-1; TLR-4, Toll-like receptor 4 .

RAGE, TLR-2 and TLR-4, of which RAGE may be the most important. The use of RAGE antagonists alone hinders ICH-induced inflammatory cell infiltration, and decreases its downstream factors IL-1 $\beta$ and MMP-9 in the brain tissue around the site of the ICH (57). As a downstream factor of HMGB1, NF- $\kappa B$ is highly sensitive to oxidative stress in the surrounding area following $\mathrm{ICH}$, and mediates downstream IL-1 $\beta$ and ICAM-1, which serve a key role in cell death following ICH, particularly in apoptosis (55).

At the end of the course of the disease, the brain begins to undergo a remodeling process, involving synaptic and vascular regeneration, which may aid in the recovery of nerve function following ICH. In this process, the progenitor cells of the subventricular region of the hippocampus migrate into the damaged brain region and differentiate into mature neurons and glial cells (59). HMGB1 serves an important role in promoting tissue recovery and remodeling at the late stage of disease. Following ICH, the expression levels of HMGB1 and VEGF are increased in surrounding brain tissue, and the inhibition of HMGB1 activity could significantly reduce the upregulation of VEGF (56). The ICH rat model induced by collagenase indicates that HMGB1 promotes angiogenesis mainly by mediating RAGE (56). In addition, HMGB1 can promote the expression of MMP-9, improve brain damage and restore nerve function (54). Therefore, when inhibiting the HMGB1-RAGE signaling pathway for ICH treatment, attention should be paid to the fact that early inhibition of this 


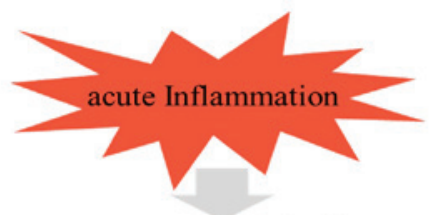

astrocytes, endothelial cells, neurons progenitor cells

migrate into the damaged

brain region

mature neurons and glial cells

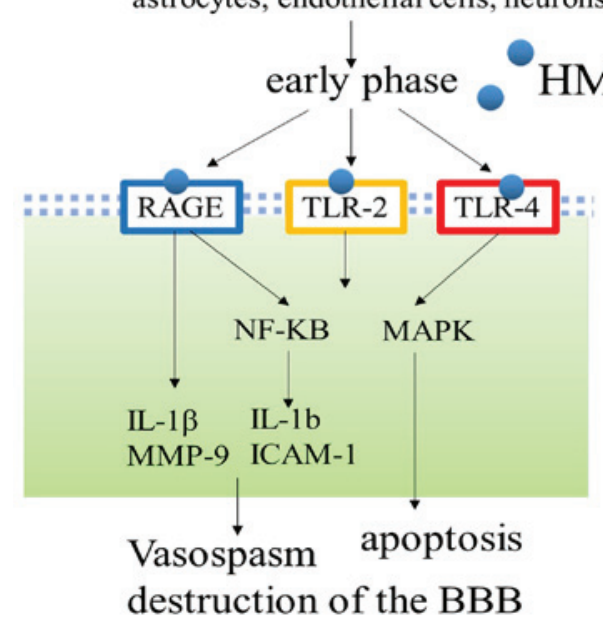

$\stackrel{\circ}{G B}$
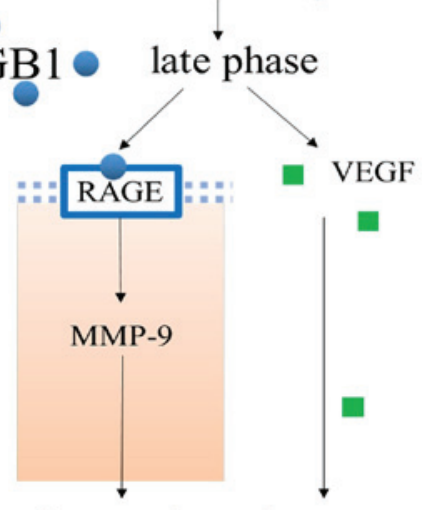

Angiogenesis and neurogenesis

Figure 3. Signaling pathway of HMGB1 in the early and late phases of cerebrovascular disease. Following acute cerebrovascular disease, under the stimulation of inflammation, astrocytes, endothelial cells and neurons can actively secrete HMGB1, while passive release occurs instantaneously upon the destruction of cellular integrity. Extracellular HMGB1 interacts with neurons, endothelial cells and glial cells to participate in BBB disruption, vasospasm and apoptosis through mediating downstream inflammatory factors, which contribute toward cerebral edema and nerve injury. On the other hand, during the late phase, HMGB1 can promote brain repair and remodeling, participating in angiogenesis and neurogenesis, contributing toward the recovery of neurological function. HMGB1, high mobility group box B1; BBB, blood brain barrier; TLR, Toll-like receptor; VEGF, vascular endothelial growth factor; NF- $\kappa \mathrm{B}$, nuclear factor- $\kappa \mathrm{B}$; MAPK, mitogen-activated protein kinase; IL, interleukin; MMP, matrix metalloproteinase; ICAM-1, intercellular cell adhesion molecule-1.

pathway can improve the treatment of $\mathrm{ICH}$, while later inhibition of this pathway could hinder the recovery of neurological function (57). These studies indicated that HMGB1 exerted different effects at different phases of intracranial hemorrhage; therefore, future studies need to focus on determining time frames.

Cerebral venous thrombosis. Cerebral venous sinus thrombosis is a relatively rare cerebrovascular disease, which accounts for $0.5-1 \%$ of the causes of stroke. A total of $50 \%$ of patients have venous cerebral infarction with a series of clinical manifestations, including headache, hemiplegia, epileptic seizures and intracranial hypertension. Its pathophysiological mechanism can be explained by the fact that, following thrombosis, the pressure in the veins and capillaries is higher and blood return is limited, leading to tissue hypoxia, which contributes toward brain edema and $\mathrm{ICH}$. The increase in venous pressure also hinders the reabsorption of the cerebrospinal fluid and further aggravates the intracranial hypertension (60). Although an increasing amount of attention has been paid to CVST in recent years, little is known regarding the exact pathogenesis and progression of the disease.

In recent years, a number of studies have demonstrated that HMGB1 may be involved in the pathogenesis of CVST (Fig. 2): i) In a study undertaken by $\mathrm{Gu}$ et al (61), the protein and mRNA expression levels of HMGB1-RAGE were upregulated in the cerebral infarction area in rats following CVST. Recombinant human soluble (rhs)-TM was added to the model, which reduced the nerve injury and infarct volume, and reduced the expression level of HMGB1-RAGE and the proinflammatory cytokines TNF- $\alpha$, IL-1 $\beta$ and IL- 6 in the ischemic penumbra; and ii) in the pathogenesis of CVST, venous return is blocked, but in view of the abundant collateral circulation of the cerebral venous system, there may be other mechanisms involved in the occurrence of cerebral edema following CVST. Nagai et al (62), demonstrated that following the occurrence of CVST, the concentration of monocyte chemoattractant protein-1 (MCP-1) increased. Meanwhile, HMGB1 could activate the expression of MCP-1 (23); therefore, HMGB1 may also be involved in the inflammatory response process of CVST in this way. A study undertaken by Yang et al (63), also demonstrated that apoptosis participated in the pathogenesis of CVST: caspase-3 served an important role in cellular apoptosis, and caspase-3 participated in the pathogenesis of CVST. Bax is a type of pro-apoptotic protein and Bcl-2 is one of the most active inhibitors of apoptosis. Bcl-2/Bax has been demonstrated to be essential for determining whether apoptosis has occurred, and the ratio of $\mathrm{Bcl}-2 / \mathrm{Bax}$ decreases significantly following the occurrence of CVST. HMGB1 can induce apoptosis via NF- $\kappa \mathrm{B}$ by activating TLR-4 (47). The results of these studies suggested that HMGB1 serves an important role in the pathogenesis of CVST.

In general, following acute cerebrovascular incident, HMGB1 interacts with neurons, endothelial cells and glial cells to participate in BBB disruption, vasospasm and apoptosis through mediating downstream inflammatory factors, which contribute toward cerebral edema and nerve injury. On the other hand, during the recovery phase, HMGB1 can promote brain repair and remodeling, contributing toward the recovery of neurological function. Therefore, targeted therapy for HMGB1 may have a positive effect on acute cerebrovascular disease (Fig. 3). 


\section{Conclusion}

Due to the diversity of acute cerebrovascular diseases and complex pathophysiological mechanisms, it is very difficult to fundamentally cure acute cerebrovascular diseases by relying on the existing treatment methods. Gene therapy has the characteristics of high specificity, high biological activity and low toxicity, and can be targeted to improve or inhibit the expression of the target gene in vivo, so as to achieve the purpose of treating a disease. HMGB1 serves an important role in promoting the inflammatory response in acute cerebrovascular diseases. Gene therapy targeting HMGB1 may achieve satisfactory results in patients with acute cerebrovascular diseases.

\section{Acknowledgements}

Not applicable.

\section{Funding}

The present study was supported by the Natural Science Foundation of Fujian Province (grant no. 2017J01323).

\section{Availability of data and materials}

All data generated or analyzed during this study are included in this published article.

\section{Authors' contributions}

SWM researched literatures, and was a major contributor in writing the manuscript. YD researched literatures and edited the manuscript. SSW reviewed the manuscript. JJG reviewed the manuscript and approved the final version. All authors read and approved the final manuscript.

\section{Ethics statement and consent to participate}

Not applicable.

\section{Patient consent for publication}

Not applicable.

\section{Competing interests}

The authors declare that they have no competing interests.

\section{References}

1. Goodwin GH, Sanders C and Johns EW: A new group of chromatin-associated proteins with a high content of acidic and basic amino acids. Eur J Biochem 38: 14-19, 1973.

2. Sohun M and Shen H: The implication and potential applications of high-mobility group box 1 protein in breast cancer. Ann Transl Med 4: 217, 2016.

3. Bertheloot D and Latz E: HMGB1, IL-1 $\alpha$, IL-33 and S100 proteins: Dual-function alarmins. Cell Mol Immunol 14: 43-64, 2017.

4. George MG, Tong X and Bowman BA: Prevalence of cardiovascular risk factors and strokes in younger adults. JAMA Neurol 74: 695-703, 2017
5. Fotakopoulos G, Tsianaka E, Fountas K, Makris D, Spyrou M and Hernesniemi J: Clipping versus coiling in anterior circulation ruptured intracranial aneurysms: A meta-analysis. World Neurosurg 104: 482-488, 2017.

6. Ilyas A, Chen CJ, Raper DM, Ding D, Buell T, Mastorakos P and Liu KC: Endovascular mechanical thrombectomy for cerebral venous sinus thrombosis: A systematic review. J Neurointerv Surg 9: 1086-1092, 2017.

7. Musumeci D, Roviello GN and Montesarchio D: An overview on HMGB1 inhibitors as potential therapeutic agents in HMGB1-related pathologies. Pharmacol Ther 141: 347-357, 2014.

8. Andersson U and Tracey KJ: HMGB1 is a therapeutic target for sterile inflammation and infection. Annu Rev Immunol 29: 139-162, 2011.

9. Yang H, Wang H, Chavan SS and Andersson U: High mobility group box protein 1 (HMGB1): The prototypical endogenous danger molecule. Mol Med 21 (Suppl 1): S6-S12, 2015.

10. Antoine DJ, Harris HE, Andersson U, Tracey KJ and Bianchi ME: A systematic nomenclature for the redox states of high mobility group box (HMGB) proteins. Mol Med 20: 135-137, 2014.

11. Andersson U, Antoine DJ and Tracey KJ: The functions of HMGB1 depend on molecular localization and post-translational modifications. J Intern Med 276: 420-424, 2014.

12. Lu B, Antoine DJ, Kwan K, Lundbäck $P$, Wähämaa $H$, Schierbeck H, Robinson M, Van Zoelen MA, Yang H, Li J, et al: JAK/STAT1 signaling promotes HMGB1 hyperacetylation and nuclear translocation. Proc Natl Acad Sci USA 111: 3068-3073, 2014.

13. Lu B, Nakamura T, Inouye K, Li J, Tang Y, Lundbäck P Valdes-Ferrer SI, Olofsson PS, Kalb T, Roth J, et al: Novel role of PKR in inflammasome activation and HMGB1 release. Nature 488: 670-674, 2012.

14. Sánchez-Giraldo R, Acosta-Reyes FJ, Malarkey CS, Saperas N, Churchill ME and Campos JL: Two high-mobility group box domains act together to underwind and kink DNA. Acta Crystallogr D Biol Crystallogr 71: 1423-1432, 2015.

15. Martinotti S, Patrone $M$ and Ranzato E: Emerging roles for HMGB1 protein in immunity, inflammation, and cancer. Immunotargets Ther 4: 101-109, 2015.

16. Kang R, Zhang Q, Zeh HJ III, Lotze MT and Tang D: HMGB1 in cancer: Good, bad, or both? Clin Cancer Res 19: 4046-4057, 2013.

17. Yanai H, Ban T, Wang Z, Choi MK, Kawamura T, Negishi H, Nakasato M, Lu Y, Hangai S, Koshiba R, et al: HMGB proteins function as universal sentinels for nucleic-acid-mediated innate immune responses. Nature 462: 99-103, 2009.

18. Franchi L, Muñoz-Planillo R and Núñez G: Sensing and reacting to microbes through the inflammasomes. Nat Immunol 13: 325-332, 2012.

19. Lu B, Wang H, Andersson U and Tracey KJ: Regulation of HMGB1 release by inflammasomes. Protein Cell 4: 163-167, 2013.

20. Yanai H, Matsuda A, An J, Koshiba R, Nishio J, Negishi H, Ikushima $\mathrm{H}$, Onoe $\mathrm{T}$, Ohdan $\mathrm{H}$, Yoshida $\mathrm{N}$, et al: Conditional ablation of HMGB1 in mice reveals its protective function against endotoxemia and bacterial infection. Proc Natl Acad Sci USA 110: 20699-20704, 2013.

21. He SJ, Cheng J, Feng X, Yu Y, Tian L and Huang Q: The dual role and therapeutic potential of high-mobility group box 1 in cancer. Oncotarget 8: 64534-64550, 2017.

22. van Beijnum JR, Buurman WA and Griffioen AW: Convergence and amplification of toll-like receptor (TLR) and receptor for advanced glycation end products (RAGE) signaling pathways via high mobility group B1 (HMGB1). Angiogenesis 11: 91-99, 2008.

23. Fiuza C, Bustin M, Talwar S, Tropea M, Gerstenberger E, Shelhamer JH and Suffredini AF: Inflammation-promoting activity of HMGB1 on human microvascular endothelial cells. Blood 101: 2652-2660, 2003.

24. Pradere JP, Dapito DH and Schwabe RF: The Yin and Yang of Toll-like receptors in cancer. Oncogene 33: 3485-3495, 2014.

25. Xi Y, Shao F, Bai XY, Cai G, Lv Y and Chen X: Changes in the expression of the Toll-like receptor system in the aging rat kidneys. PLoS One 9: e96351, 2014.

26. Yang G, Zhang L, Ma L, Jiang R, Kuang G, Li K, Tie H, Wang B, Chen X, Xie T, et al: Glycyrrhetinic acid prevents acetaminophen-induced acute liver injury via the inhibition of CYP2E1 expression and HMGB1-TLR4 signal activation in mice. Int Immunopharmacol 50: 186-193, 2017.

27. Tian J, Avalos AM, Mao SY, Chen B, Senthil K, Wu H, Parroche P, Drabic S, Golenbock D, Sirois C, et al: Toll-like receptor 9-dependent activation by DNA-containing immune complexes is mediated by HMGB1 and RAGE. Nat Immunol 8: 487-496, 2007. 
28. Hiraku Y, Guo F, Ma N, Yamada T, Wang S, Kawanishi S and Murata M: Multi-walled carbon nanotube induces nitrative DNA damage in human lung epithelial cells via HMGB1-RAGE interaction and Toll-like receptor 9 activation. Part Fibre Toxicol 13: 16, 2016.

29. Das N, Dewan V, Grace PM, Gunn RJ, Tamura R, Tzarum N, Watkins LR, Wilson IA and Yin H: HMGB1 activates proinflammatory signaling via TLR5 leading to allodynia. Cell Reports 17 1128-1140, 2016.

30. Magna M and Pisetsky DS: The role of HMGB1 in the pathogenesis of inflammatory and autoimmune diseases. Mol Med 20: 138-146, 2014

31. Godinho J, de Oliveira RMW, de Sa-Nakanishi AB, Bacarin CC Huzita CH, Longhini R, Mello JCP, Nakamura CV, Previdelli IS, Dal Molin Ribeiro MH, et al: Ethyl-acetate fraction of Trichilia catigua restores long-term retrograde memory and reduces oxidative stress and inflammation after global cerebral ischemia in rats. Behav Brain Res 337: 173-182, 2018.

32. Tsukagawa T, Katsumata R, Fujita M, Yasui K, Akhoon C, Ono K, Dohi K and Aruga T: Elevated serum high-mobility group box-1 protein level Is associated with poor functional outcome in ischemic stroke. J Stroke Cerebrovasc Dis 26: 2404-2411, 2017.

33. Qiu J, Nishimura M, Wang Y, Sims JR, Qiu S, Savitz SI Salomone $S$ and Moskowitz MA: Early release of HMGB-1 from neurons after the onset of brain ischemia. J Cereb Blood Flow Metab 28: 927-938, 2008

34. Shichita T, Sakaguchi R, Suzuki M and Yoshimura A Post-ischemic inflammation in the brain. Front Immunol 3: 132, 2012.

35. Zhang J, Takahashi HK, Liu K, Wake H, Liu R, Maruo T, Date I, Yoshino T, Ohtsuka A, Mori S, et al: Anti-high mobility group box-1 monoclonal antibody protects the blood-brain barrier from ischemia-induced disruption in rats. Stroke 42: 1420-1428, 2011.

36. Li WA, Moore-Langston S, Chakraborty T, Rafols JA, Conti AC and Ding Y: Hyperglycemia in stroke and possible treatments. Neurol Res 35: 479-491, 2013.

37. Zhang J, Wu Y, Weng Z, Zhou T, Feng T and Lin Y: Glycyrrhizin protects brain against ischemia-reperfusion injury in mice through HMGB1-TLR4-IL-17A signaling pathway. Brain Res 1582: 176-186, 2014.

38. Gong G, Xiang L, Yuan L, Hu L, Wu W, Cai L, Yin L and Dong H: Protective effect of glycyrrhizin, a direct HMGB1 inhibitor, on focal cerebral ischemia/reperfusion-induced inflammation, oxidative stress, and apoptosis in rats. PLoS One 9: e89450, 2014

39. Umahara T, Uchihara T, Hirokawa K, Hirao K, Shimizu S, Hashimoto T, Terasi H and Hanyu H: Time-dependent and lesion-dependent HMGB1-selective localization in brains of patients with cerebrovascular diseases. Histol Histopathol 33: 215-222, 2018

40. Chen JY, Yu Y, Yuan Y, Zhang YJ, Fan XP, Yuan SY, Zhang JC and Yao SL: Enriched housing promotes post-stroke functional recovery through astrocytic HMGB1-IL-6-mediated angiogenesis. Cell Death Discov 3: 17054, 2017.

41. Wu X, Liu S, Hu Z, Zhu G, Zheng G and Wang G: Enriched housing promotes post-stroke neurogenesis through calpain 1-STAT3/HIF-1 $\alpha /$ VEGF signaling. Brain Res Bull 139: 133-143, 201841, 2018

42. Zhang D, Wu W, Yan H, Jiang T, Liu M, Yu Z, Li H and Hang C: Upregulation of HMGB1 in wall of ruptured and unruptured human cerebral aneurysms: Preliminary results. Neurol Sci 37: 219-226, 2016.

43. Chalouhi N, Ali MS, Jabbour PM, Tjoumakaris SI, Gonzalez LF, Rosenwasser RH, Koch WJ and Dumont AS: Biology of intracranial aneurysms: Role of inflammation. J Cereb Blood Flow Metab 32: 1659-1676, 2012

44. Aoki T, Kataoka H, Nishimura M, Ishibashi R, Morishita R and Miyamoto S: Regression of intracranial aneurysms by simultaneous inhibition of nuclear factor- $\kappa \mathrm{B}$ and Ets with chimeric decoy oligodeoxynucleotide treatment. Neurosurgery 70 : 1534-1543, discussion 1543, 2012

45. Bianchi ME and Manfredi AA: High-mobility group box 1 (HMGB1) protein at the crossroads between innate and adaptive immunity. Immunol Rev 220: 35-46, 2007.

46. Przybycien-Szymanska MM and Ashley WW Jr: Biomarker discovery in cerebral vasospasm after aneurysmal subarachnoid hemorrhage. J Stroke Cerebrovasc Dis 24: 1453-1464, 2015.
47. Chang CZ, Wu SC, Kwan AL and Lin CL: Rhinacanthin-C, A fat-soluble extract from Rhinacanthus nasutus, modulates high-mobility group box 1-related neuro-inflammation and subarachnoid hemorrhage-induced brain apoptosis in a rat model. World Neurosurg 86: 349-360, 2016.

48. Zhao XD, Mao HY, Lv J and Lu XJ: Expression of high-mobility group box-1 (HMGB1) in the basilar artery after experimental subarachnoid hemorrhage. J Clin Neurosci 27: 161-165, 2016.

49. Li H, Wu W, Sun Q, Liu M, Li W, Zhang XS, Zhou ML and Hang $\mathrm{CH}$ : Expression and cell distribution of receptor for advanced glycation end-products in the rat cortex following experimental subarachnoid hemorrhage. Brain Res 1543: 315-323, 2014.

50. Zhang XS, Li W, Wu O, Wu LY, Ye ZN, Liu JP, Zhuang Z, Zhou ML, Zhang $\mathrm{X}$ and Hang $\mathrm{CH}$ : Resveratrol attenuates acute inflammatory injury in experimental subarachnoid hemorrhage in rats via inhibition of TLR4 pathway. Int J Mol Sci 17: 17, 2016.

51. Hendrix P, Foreman PM, Harrigan MR, Fisher WSR III, Vyas NA, Lipsky RH, Lin M, Walters BC, Tubbs RS, Shoja MM, et al: Impact of high-mobility group box 1 polymorphism on delayed cerebral ischemia after aneurysmal subarachnoid hemorrhage. World Neurosurg 101: 325-330, 2017.

52. Tian X, Sun L, Feng D, Sun Q, Dou Y, Liu C, Zhou F, Li H, Shen H, Wang Z, et al: HMGB1 promotes neurovascular remodeling via Rage in the late phase of subarachnoid hemorrhage. Brain Res 1670: 135-145, 2017.

53. Qureshi AI, Mendelow AD and Hanley DF: Intracerebral haemorrhage. Lancet 373: 1632-1644, 2009.

54. Lei C, Wu B, Cao T, Zhang S and Liu M: Activation of the high-mobility group box 1 protein-receptor for advanced glycation end-products signaling pathway in rats during neurogenesis after intracerebral hemorrhage. Stroke 46: 500-506, 2015.

55. Wang YX, Yan A, Ma ZH, Wang Z, Zhang B, Ping JL, Zhu JS, Zhou Y and Dai L: Nuclear factor- $\mathrm{KB}$ and apoptosis in patients with intracerebral hemorrhage. J Clin Neurosci 18: 1392-1395, 2011

56. Lei C, Zhang S, Cao T, Tao W, Liu M and Wu B: HMGB1 may act via RAGE to promote angiogenesis in the later phase after intracerebral hemorrhage. Neuroscience 295: 39-47, 2015.

57. Li D, Lei C, Zhang S, Zhang S, Liu M and Wu B: Blockade of high mobility group box-1 signaling via the receptor for advanced glycation end-products ameliorates inflammatory damage after acute intracerebral hemorrhage. Neurosci Lett 609: 109-119, 2015.

58. Wang D, Liu K, Wake H, Teshigawara K, Mori S and Nishibori M: Anti-high mobility group box-1 (HMGB1) antibody inhibits hemorrhage-induced brain injury and improved neurological deficits in rats. Sci Rep 7: 46243, 2017.

59. Shang J, Deguchi K, Ohta Y, Liu N, Zhang X, Tian F, Yamashita T, Ikeda Y, Matsuura T, Funakoshi H, et al: Strong neurogenesis, angiogenesis, synaptogenesis, and antifibrosis of hepatocyte growth factor in rats brain after transient middle cerebral artery occlusion. J Neurosci Res 89: 86-95, 2011.

60. Sharma KM and Ahn J: Cerebral venous sinus thrombophlebitis as a complication of acute otitis media. J Emerg Med 48: e9-e13, 2015.

61. Gu JJ, Chen JB, Zhang JH, Zhang H and Wang SS: Recombinant human soluble thrombomodulin protects against brain injury in a CVST rat model, via downregulation of the HMGB1-RAGE axis. Mol Med Rep 14: 5217-5222, 2016.

62. Nagai M, Terao S, Yilmaz G, Yilmaz CE, Esmon CT, Watanabe E and Granger DN: Roles of inflammation and the activated protein $\mathrm{C}$ pathway in the brain edema associated with cerebral venous sinus thrombosis. Stroke 41: 147-152, 2010.

63. Yang H, Meng Z, Zhang C, Zhang P and Wang Q: Establishing a new rat model of central venous sinus thrombosis and analyzing its pathophysiological and apoptotic changes. J Neurosci Methods 203: 130-135, 2012

This work is licensed under a Creative Commons Attribution-NonCommercial-NoDerivatives 4.0 International (CC BY-NC-ND 4.0) License. 